\title{
A simple analytical model for predicting the detectable ion current in ion mobility spectrometry using corona discharge ionization sources
}

\author{
Ansgar T. Kirk, Tim Kobelt, Hauke Spehlbrink, Stefan Zimmermann \\ Leibniz Universität Hannover, Institute of Electrical Engineering and Measurement Technology, \\ Department of Sensors and Measurement Technology, Appelstr. 9A, 30167 Hannover, Germany
}

\begin{abstract}
Corona discharge ionization sources are often used in ion mobility spectrometers (IMS) when a nonradioactive ion source with high ion currents is required. Typically, the corona discharge is followed by a reaction region where analyte ions are formed from the reactant ions. In this work, we present a simple yet sufficiently accurate model for predicting the ion current available at the end of this reaction region when operating at reduced pressure as in High Kinetic Energy Ion Mobility Spectrometers (HiKE-IMS) or most IMS-MS instruments. It yields excellent qualitative agreement with measurement results and is even able to calculate the ion current within an error of $15 \%$. Additional interesting findings of this model are the ion current at the end of the reaction region being independent from the ion current generated by the corona discharge and the ion current in High Kinetic Energy Ion Mobility Spectrometers (HiKE-IMS) growing quadratically when scaling down the length of the reaction region.
\end{abstract}

\section{Introduction}

Many ion mobility spectrometers (IMS) use corona discharge ionization sources followed by a reaction region as a non-radioactive ionization source [1-7], a design first introduced with coronaspray IMS by Hill et al. [8]. The major disadvantage of a corona discharge ionization source is the different reaction chemistry compared to radioactive ionization sources, especially in the negative mode [9-11]. Nevertheless, a corona discharge ionization source is non-radioactive and able to generate significant ion currents in the range of several tens of microamperes at the source, compared to a few hundred picoamperes when using radioactive ionization sources. Thus, corona discharge is an interesting ionization source for many applications, especially for HiKE-IMS [12; 13], in which a control over the reaction kinetics is possible and high ion currents are necessary to maintain good sensitivity at reduced pressures. However, the ion current available at the ion shutter and not at the source is important for the sensitivity of an ion mobility spectrometer. Increasing this current increases the amount of ions that can be transferred into the drift region and thus the signal-to-noise-ratio of peaks likewise, regardless of losses inside the drift region.

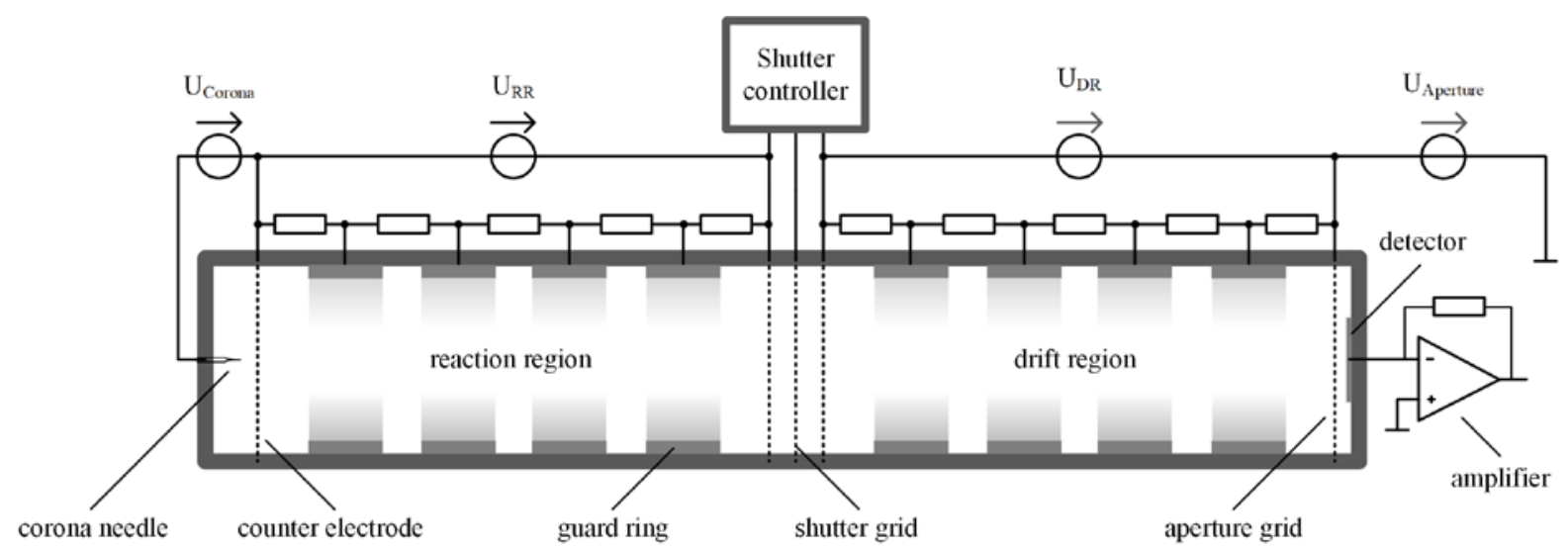

Figure 1: Schematic setup of an IMS with a corona discharge ionization source. 
The general setup of an ion mobility spectrometer with a corona discharge ionization source is shown in Figure 1, consisting of a corona needle, an optional counter electrode, a reaction region, a drift region, an ion shutter separating the reaction region from the drift region, and a detector. Ions are generated in the discharge region at the corona needle tip and then travel towards the ion shutter under the influence of the electric field applied to the reaction region. While travelling through the reaction region, the ion beam expands radially due to diffusion and coulomb repulsion, causing ions to be lost at the ring electrodes. At the ion shutter, the ion beam is chopped into small segments (ion packets) containing reactant and analyte ions that are subsequently separated inside the drift region. The resulting ion current peaks at the detector are then amplified by a transimpedance amplifier and recorded over time, leading to the ion mobility spectrum. It is noteworthy that this setup is similar to those used with electrospray or Nickel-63 ionization sources and thus the model developed here may be transferred to other ion sources containing a reaction region.

\section{Analytical model}

In order to describe the processes involved, a simple analytical model is often the most applicable approach, as it reveals the main relationships and can provide good estimations with little effort. As a corona discharge is an ionization source with small volume, being the plasma at the corona needle tip, but high ion current, often several microamperes, coulomb repulsion can be expected to be the major effect causing ion beam expansion. Thus, the simplest approach is a model just considering coulomb expansion of the ion beam while the ions travel through the reaction region.

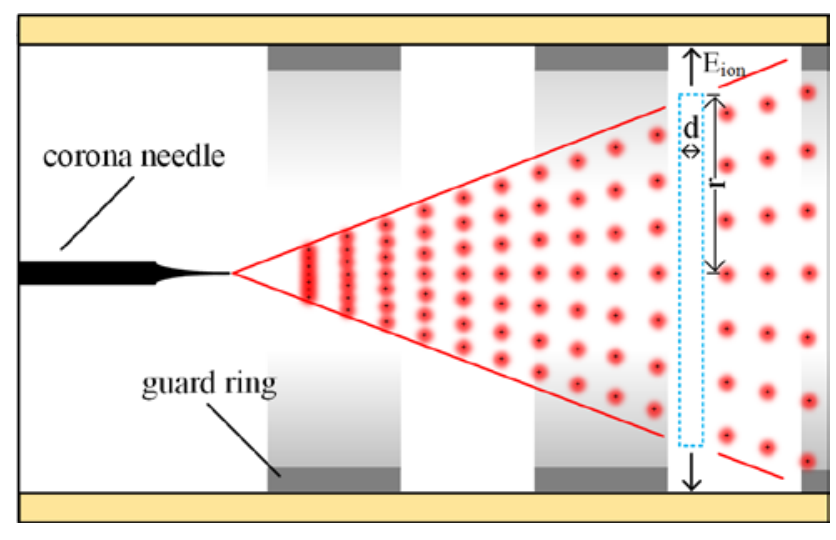

Figure 2: Depiction of the ion beam expanding from the corona needle.

Let us consider an infinitesimally thin slice of this ion beam as indicated by the blue rectangle in Figure 2 and apply Gauss' Law. This ion slice travels in-between two adjacent slices with comparable ion density and diameter, as the ion beam expands only gradually. Thus, the electric fields of adjacent ion slices in the direction of travel and vice versa must compensate each other, causing the electric field of the charge to extend only in radial direction. This radial electric field at the circumference of the ion beam $E_{\text {ion }}$ can be derived from Gauss' Law by dividing the charge inside the infinitesimally thin slice by its radial surface area and the free space permittivity $\varepsilon_{0}$ as shown by Eq. 1 . The charge per length is given by the ratio of the corona ion current $I_{\text {cor }}$ to the velocity of the ion slice $\mathrm{v}_{\text {ion. }}$. This can be seen from the current being the amount of charge passing per time and the velocity being the length covered per time. The velocity can be expressed through the ion mobility $K$ and the electric field strength applied to the reaction region $E_{R R}$. The radial surface area per length is simply $2 \pi$ times the radius of the ion beam $r$.

$$
E_{\text {ion }}=\frac{I_{\text {cor }} d}{v_{\text {ion }}} \frac{1}{2 \pi r d} \frac{1}{\varepsilon_{0}}=\frac{I_{\text {cor }}}{2 \pi r \varepsilon_{0} K E_{R R}}
$$


In the next step, the radial motion of the ion slice's circumference will be considered. It is the time derivative of the radius of the ion beam and depends on the ions' mobility and the radial field generated by the ions as given by Eq. 2 .

$$
\frac{d r}{d t}=K E_{\text {ion }}=\frac{I_{\text {cor }}}{2 \pi r \varepsilon_{0} E_{R R}}
$$

Solving this differential equation by separation of variables leads to Eq. 3, which specifies the radius of the ion beam as a function of time $t$.

$$
r(t)=\sqrt{\frac{I_{\text {cor }} t}{\pi \varepsilon_{0} E_{R R}}}
$$

Assuming that the ion current is equally distributed across this radius leads to Eq. 4 specifying the current density $J$ inside the ion beam. The calculated radius of the ion beam may extend beyond the actual radius of the reaction region. In this case, the outer ions are discharged at the ring electrodes. However, as the detector usually has a smaller diameter than the reaction and drift region, this should not affect the ions actually being detected. It is especially noteworthy that the corona discharge current cancels out, as a larger ion current leads to equally larger repulsion.

$$
J=\frac{I_{c o r}}{\pi r^{2}}=\frac{\varepsilon_{0} E_{R R}}{t_{R R}}
$$

The time $t_{R R}$ spent inside the reaction region with length $L_{R R}$ can be calculated using the reduced mobility $K_{0}$, leading to Eq. 5 . The first factor can be expressed through a constant c $=\varepsilon_{0} \mathrm{~K}_{0} \mathrm{~N}_{0}$, while the second factor can be rewritten to include the reduced field strength $E / N$. This is a more useful notation, as practically all gas phase ion processes depend on the reduced field strength and not on the electric field strength.

$$
J=\varepsilon_{0} K_{0} N_{0} \frac{E_{R R}^{2}}{N L_{R R}}=c\left(\frac{E_{R R}}{N}\right)^{2} \frac{N}{L_{R R}}
$$

The interpretation of Eq. 5 leads to several important connections. First, as already noted, the ion current at the end of the reaction region is independent from the corona discharge current. This will of course only hold for corona discharge currents high enough to lead to an ion beam larger than the detector through expansion by coulomb repulsion. As soon as the reduced field strength is high enough to guide all ions to the detector, the ion current obviously cannot rise any further. Second, the ion current grows quadratically with increasing reduced field strength, as the ion beam has less time to expand by coulomb repulsion and the higher ion velocity reduces the ion density. This agrees well with the experimental results of Tabrizchi et al. [1] and An et al. [14], who both observed a quadratic increase in ion current with increasing applied voltage. Third, in HiKE-IMS, where the reduced field strength $E / N$ is an experimental parameter used to control the ion chemistry, the pressure inside the device is inversely proportional to the length at a constant available voltage [15]. Thus, reducing the length and increasing the pressure in HiKE-IMS would result in a quadratically increasing ion current at the same reduced field strength $E / N$.

So far, only the ion transmission through the reaction region has been considered. However, for a complete model the transmission through the ion shutter grids also has to be taken into account. On the one hand, this of course depends on the opening duration and mobility discrimination. These are mostly operational parameters of the ion shutter. On the other hand, ion shutters in the open state are practically metallic grids. The transmission through such grids also leads to losses, causing the ion current transmitted through the ion shutter even in the open state to be lower than the current arriving at the ion shutter. A good estimation developed in previous models is the transmission being the product of a transparency factor $c_{\text {trans }}$ (which is not the optical transparency) and the ratio of the 
electric field behind the ion shutter to the electric field in front of the ion shutter $[1 ; 16 ; 17]$. This leads to Eq. 6. It is assumed that the ion loss when passing the ion shutter minimizes further ion beam expansion by coulomb repulsion inside the drift region due to reduced ion density. Thus, all ions transmitted through the ion shutter would arrive at the detector.

$$
J_{\text {det }}=c_{\text {trans }} \frac{E_{D R}}{E_{R R}} J=c_{\text {trans }} c \frac{E_{R R}}{N} \frac{E_{D R}}{N} \frac{N}{L_{R R}}
$$

\section{Experimental setup}

Two different experimental setups were used to verify Eq. 5 and Eq. 6. First, the detector was placed directly in front of the ion shutter in order to measure the ion current arriving at the ion shutter at the end of the reaction region under different experimental conditions. Second, a drift tube was added behind the ion shutter to measure the ion current after ion loss caused by the ion shutter.

The corona voltage was supplied by a FUG HCP-35-20000 power supply operated in currentcontrolled mode. All other voltages were supplied by homemade power supplies. The setup was evacuated by a Pfeiffer MVP 040 membrane pump and the resulting pressure was monitored using a Pfeiffer CMR 362 pressure gauge. The ion current was amplified using a FEMTO DLPCA-200 current amplifier set to a bandwidth of $10 \mathrm{~Hz}$, as only DC currents need to be measured.

Table 1: Operational parameters

\begin{tabular}{|c|c|}
\hline Parameter & Value \\
\hline Reaction region length & $41,77,91,127 \mathrm{~mm}$ \\
\hline Drift region length & $41,77 \mathrm{~mm}$ \\
\hline Corona discharge current & $5-30 \mu \mathrm{A}$ \\
\hline Reaction region reduced field & $20-125 \mathrm{Td}$ \\
\hline Drift region reduced field & $20-150 \mathrm{Td}$ \\
\hline Operating pressure & $6.5-80 \mathrm{mbar}$ \\
\hline Drift gas flow & $\begin{array}{c}10 \mathrm{ml} / \mathrm{min} \\
\left(\text { at } 1013 \mathrm{mbar} / 20^{\circ} \mathrm{C}\right)\end{array}$ \\
\hline Drift gas dew point & $\begin{array}{c}-90^{\circ} \mathrm{C} \\
\left(90 \mathrm{ppb}_{\mathrm{v}} \text { water vapor }\right. \\
\mathrm{concentration}^{\circ}\end{array}$ \\
\hline Operating temperature & $25^{\circ} \mathrm{C}$ \\
\hline
\end{tabular}

\section{Results and Discussion}

The left-hand side of Figure 3 depicts the measured ion current density at the end of the reaction region for six different corona currents. For all measurements, a reaction region length of $77 \mathrm{~mm}$ and a pressure of 15 mbar were used. The reduced field strength was swept from $30 \mathrm{Td}$ to $125 \mathrm{Td}$. No analytes were introduced and thus all ions correspond to the reactant ion species generated by the corona discharge. The x-axis is scaled according to Eq. 5, which should align all measurements onto a single straight line through the origin. It can be seen that all measurements agree very well with Eq. 5 without any dependence on the corona discharge currents. Most likely, there is a minimum corona discharge current required for the presented model to be valid, however, lower corona discharge currents could not be used as the power supply was not able to run a stable corona discharge at lower currents. 

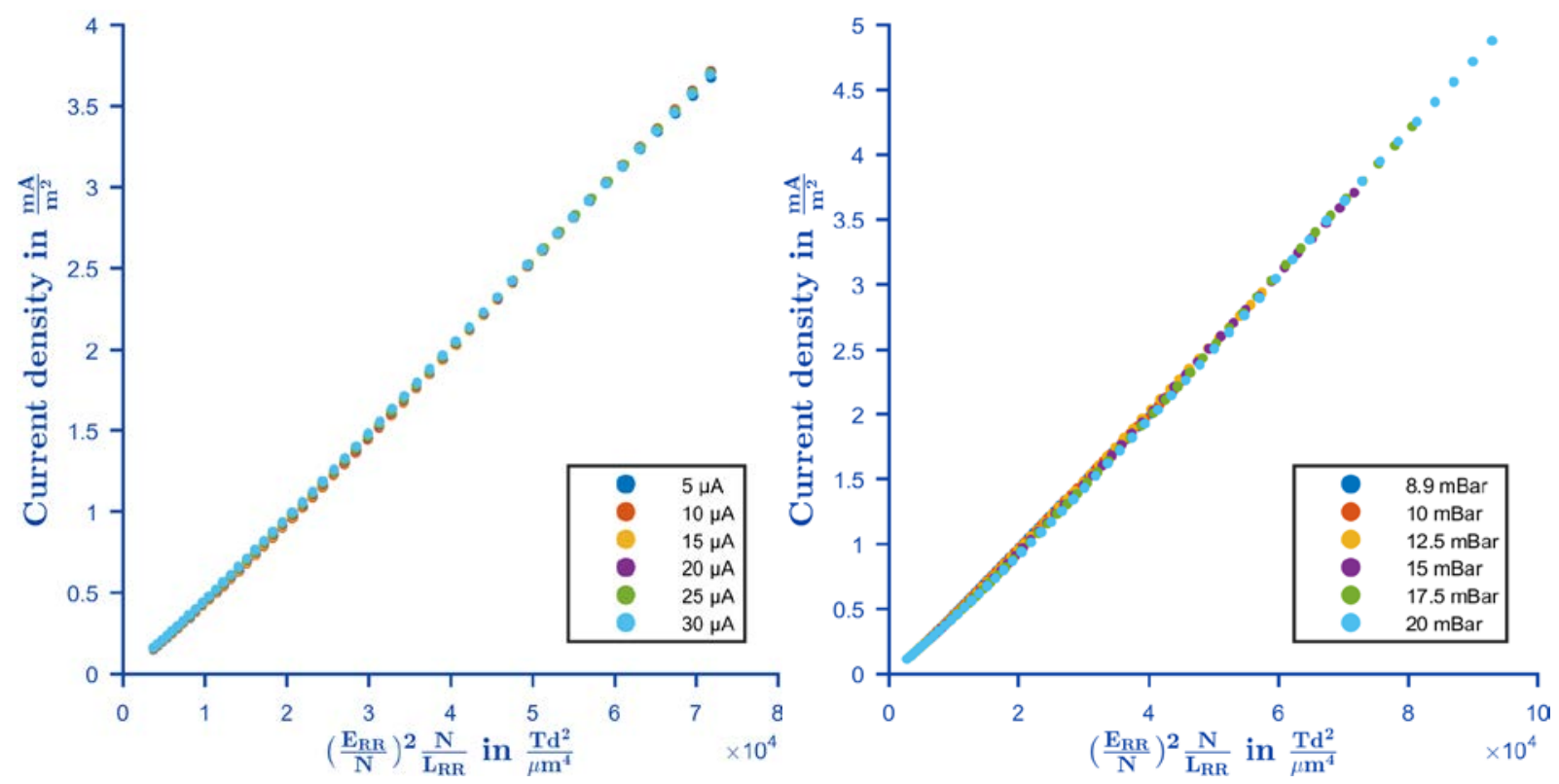

Figure 3: Current density at the end of the reaction region for different corona discharge currents (left) and different pressures (right).

Furthermore, measurements at six different pressures were compared as shown on the right-hand side of Figure 3. A reaction region length of $77 \mathrm{~mm}$ and a constant corona discharge current of $15 \mu \mathrm{A}$ were used. The reduced field strength was swept from $25 \mathrm{Td}$ to $120 \mathrm{Td}$. Again, the normalization remains valid across the whole measurement range.

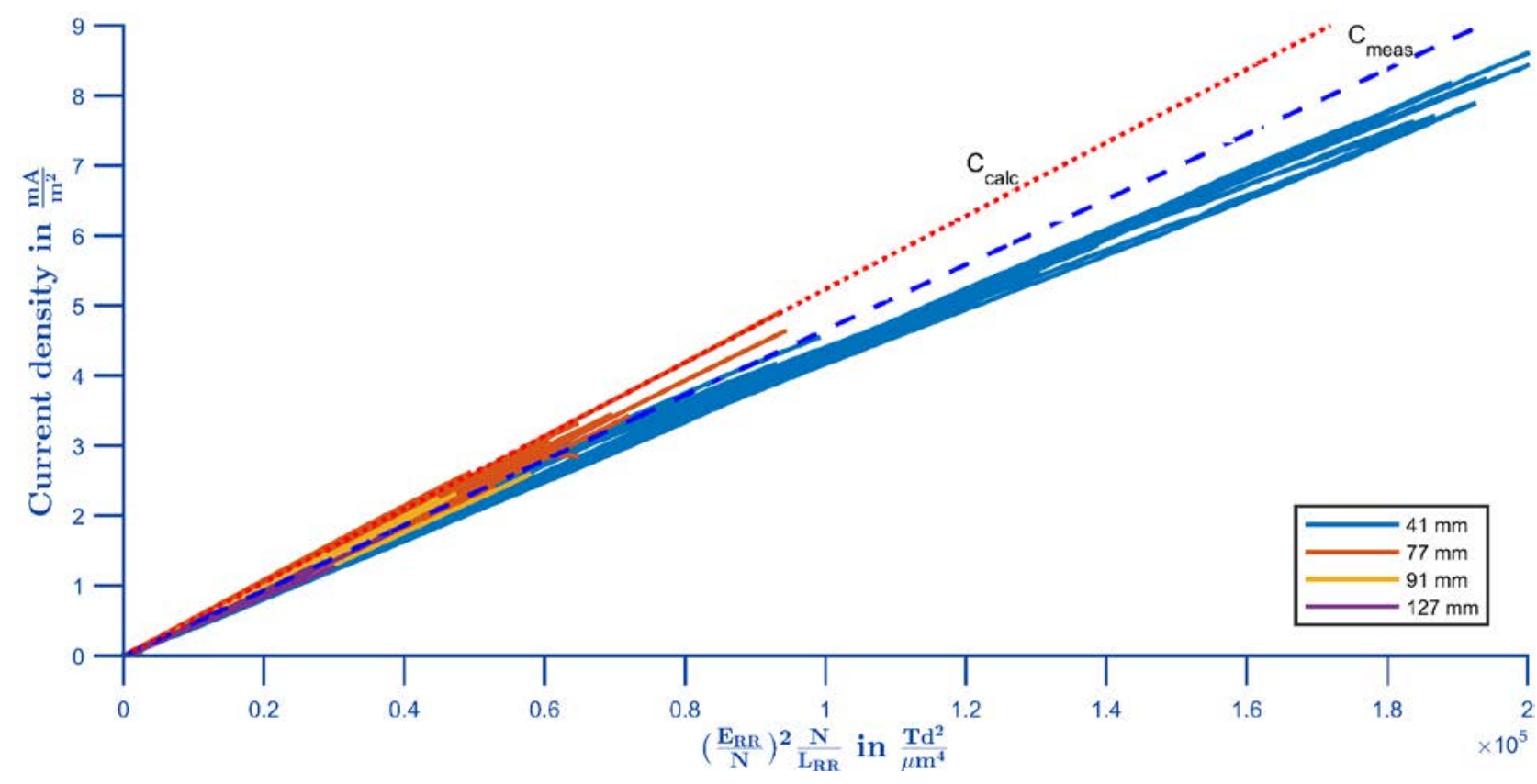

Figure 4: Current density at the end of the reaction region for different lengths, pressures and corona currents. The blue dashed line shows a fit to all measurement values, while the dotted red line is calculated from eq. 5.

Having verified Eq. 5 with respect to the corona discharge current and pressure, all measurements were repeated using four different reaction region lengths. The results are shown in Figure 4. Measurements with the same reaction region length have the same color. In total, 151 measurements are shown in Figure 4. While slight variations between all the different measurement parameter permutations occur, the agreement between all the measurements is still excellent.

Furthermore, both a linear fit through all the measurements $\left(\mathrm{c}_{\text {meas }}\right)$ and a graph $\left(\mathrm{c}_{\text {calc }}\right)$ calculated according to Eq. 5 with a reduced mobility of $\mathrm{K}_{0}=2.2 \mathrm{~cm}^{2} \mathrm{~V}^{-1} \mathrm{~s}^{-1}$ [12] are shown. The fitted constant is 4.65E $+10 \mathrm{AV}^{-2} \mathrm{~m}^{-2}$, while the calculated value is $5.23 \mathrm{E}+10 \mathrm{AV}^{-2} \mathrm{~m}^{-2}$, which is an error of just $12 \%$. 
The spread of the measured values around the linear fit is also within $\pm 15 \%$. Considering the simplicity of the used model, the agreement is impressive. Nevertheless, one factor not taken into account so far is the ion mobility changing at high reduced field strengths.

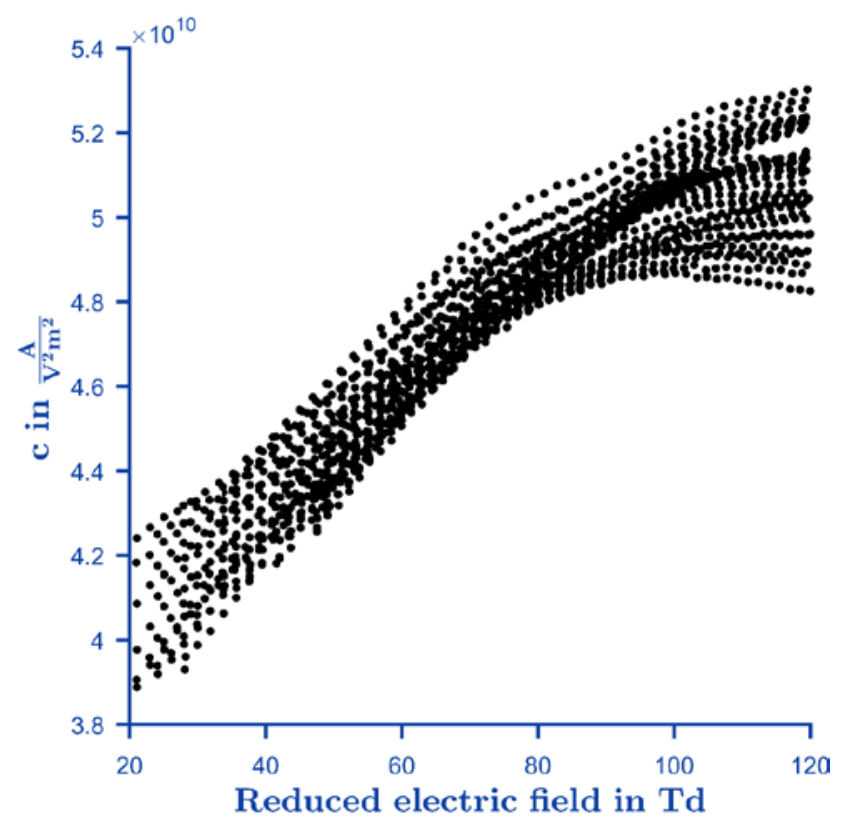

Figure 5: Different values of the constant c calculated from each single measurement in Figure 4 as a function of the reduced field strength.

Figure 5 shows the values of the constant c calculated from each single measurement from Figure 4 and plotted against the reduced field strength. It can be seen that all values fit more or less to a common function, indicating that the error is indeed a function of the reduced field strength. The most likely candidate is, as stated before, the field dependence of the reduced mobility.

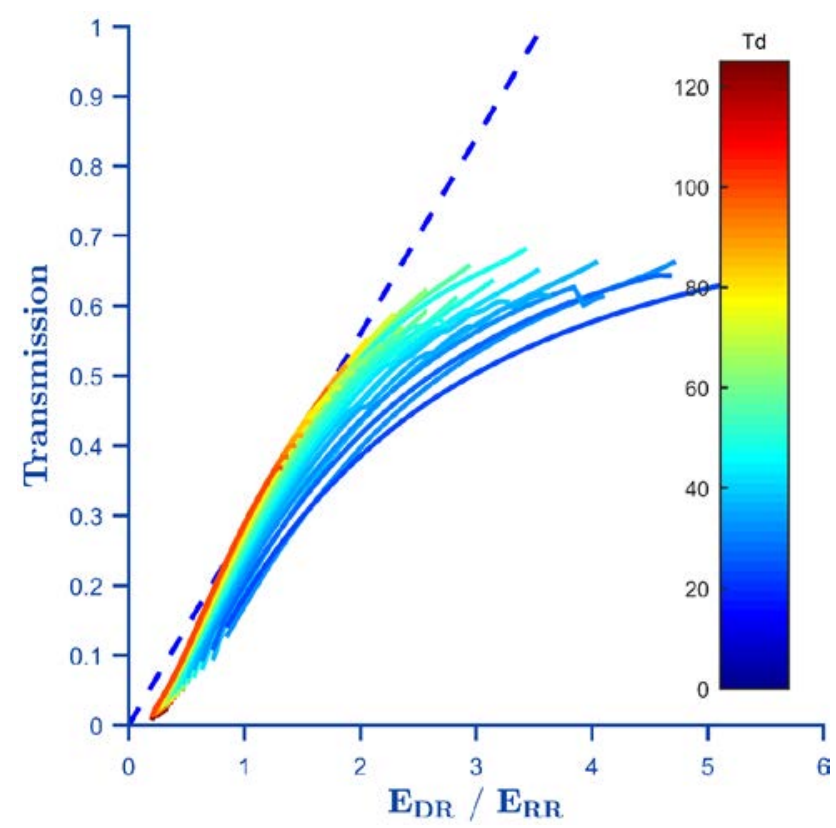

Figure 6: Relative ion transmission through the open ion shutter depending on the ratio of the reduced field strength in the drift region to the reduced field strength in the reaction region. The blue dashed line is an estimation for a linear ion transmission.

So far, only the ion current at the end of the reaction region was studied. Now, these measured current values were compared with measured current values at the end of the drift region under the same conditions. The ratio of both ion currents, corresponding to the ion transmission through the ion shutter, is shown in Figure 6. As predicted, the ion transmission through the ion shutter initially increases linearly with the ratio of the reduced field strength in the drift region to the reduced field 
strength in the reaction region. However, for higher values of $\mathrm{E}_{\mathrm{DR}} / \mathrm{E}_{\mathrm{RR}}$, the transmission starts to saturate. Nevertheless, Eq. 6 should predict the ion current density at the detector up to high ratios of $\mathrm{E}_{\mathrm{DR}} / \mathrm{E}_{\mathrm{RR}}$ with an acceptable error.

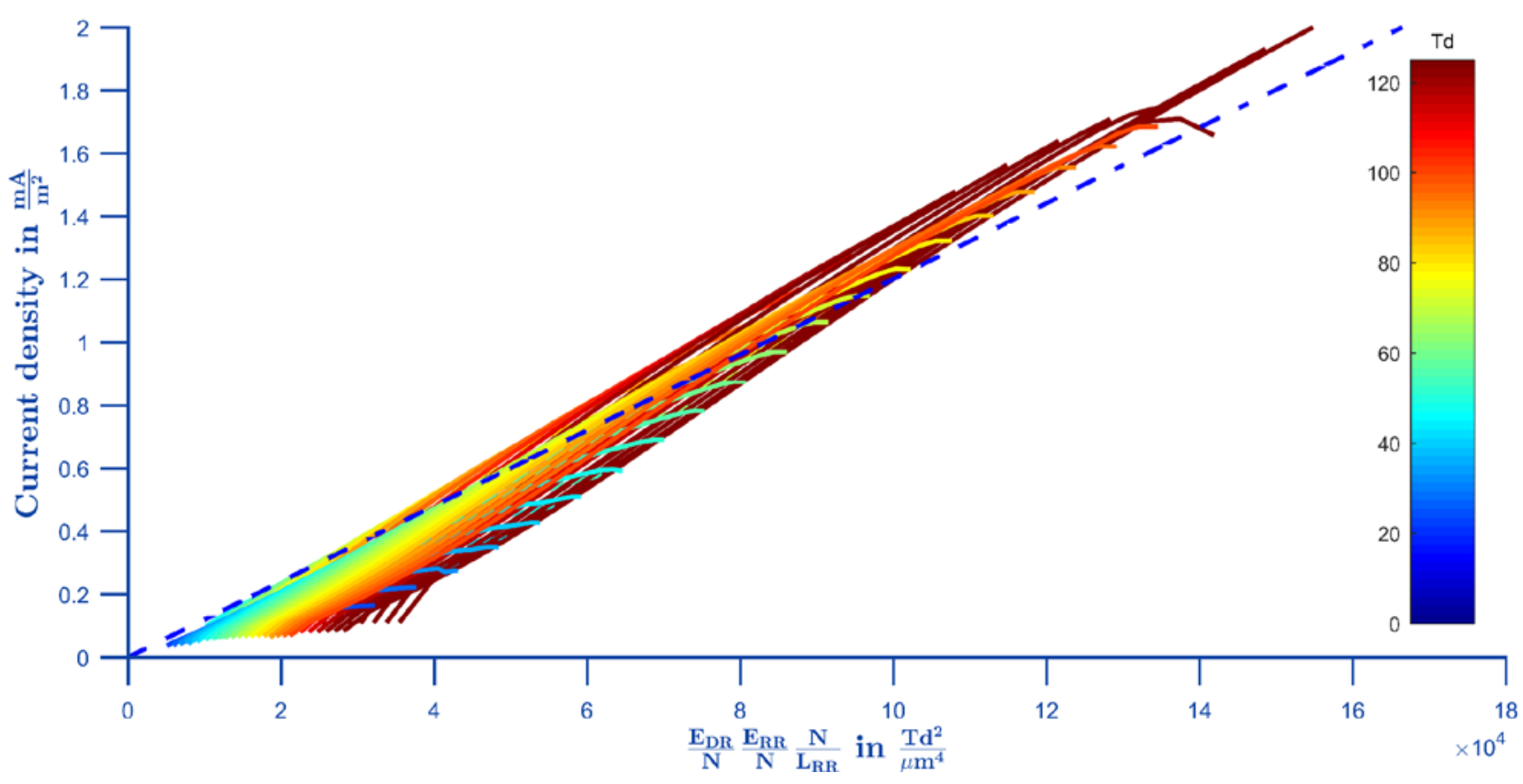

Figure 7: Current density at the detector for different pressures and corona currents. The blue dashed line is a linear fit to all measurement data.

As shown in Figure 7, this is indeed the case. When normalized according to Eq. 6, all measurements show low deviation from the linear fit. Thus, even the transmission through the ion shutter is predicted reasonably by the model.

\section{Conclusion}

In this paper, we present a simple yet sufficiently accurate analytical model for predicting the ion current of ion mobility spectrometers operated at low pressure, both at the ion shutter at the end of the reaction region and at the detector at the end of the drift region. These findings apply primarily to High Kinetic Energy Ion Mobility Spectrometry (HiKE-IMS) or most IMS-MS instruments with ionization sources that require a certain reaction region length. In particular, the model considers the ion current generated by a corona discharge source, ion losses in the reaction region due to ion beam expansion caused by coulomb repulsion and ion losses at the ion shutter. Both qualitative and quantitative predictions are possible, being a useful tool for instrument design. Especially for the optimization of HiKE-IMS, the model gives important information, as it predicts a quadratically growing ion current with system miniaturization.

\section{Acknowledgments}

Funded by the Deutsche Forschungsgemeinschaft (DFG, German Research Foundation) - ZI 1288/71.

We thank Maria Allers and Florian Schlottmann for their help during this work.

\section{References}


1. Tabrizchi M, Khayamian T, Taj N: Design and optimization of a corona discharge ionization source for ion mobility spectrometry. Rev. Sci. Instrum. 71, 2321-2328 (2000)

2. Borsdorf H, Schelhorn H, Flachowsky J, Döring H-R, Stach J: Corona discharge ion mobility spectrometry of aliphatic and aromatic hydrocarbons. Analytica chimica acta 403, 235-242 (2000)

3. Hill CA, Thomas, C. L. P.: A pulsed corona discharge switchable high resolution ion mobility spectrometer-mass spectrometer. Analyst 128, 55-60 (2003)

4. Tabrizchi M, Abedi A: A novel electron source for negative ion mobility spectrometry. Int. J. Mass Spectrom. 218, 75-85 (2002)

5. Ross SK, Bell AJ: Reverse flow continuous corona discharge ionisation applied to ion mobility spectrometry. Int. J. Mass Spectrom. 218, L1-L6 (2002)

6. Roscioli KM, Davis E, Siems WF, Mariano A, Su W, Guharay SK, Hill HH: Modular ion mobility spectrometer for explosives detection using corona ionization. 83, 5965-5971 (2011)

7. Sabo M, Matejčík Š: Corona discharge ion mobility spectrometry with orthogonal acceleration time of flight mass spectrometry for monitoring of volatile organic compounds. Anal. Chem. 84, 53275334 (2012)

8. Shumate $\mathrm{CB}$, Hill $\mathrm{HH}$ : Coronaspray nebulization and ionization of liquid samples for ion mobility spectrometry. 61, 601-606 (1989)

9. Borsdorf H, Nazarov EG, Eiceman GA: Atmospheric pressure chemical ionization studies of nonpolar isomeric hydrocarbons using ion mobility spectrometry and mass spectrometry with different ionization techniques. J. Am. Soc. Mass Spectrom. 13, 1078-1087 (2002)

10. Borsdorf $\mathrm{H}$, Nazarov EG, Eiceman GA: Atmospheric pressure ionization and gas phase ion mobility studies of isomeric dihalogenated benzenes using different ionization techniques. Int. J. Mass Spectrom. 232, 117-126 (2004)

11. Crawford $\mathrm{CL}$, Hill HH: Comparison of reactant and analyte ions for ${ }^{63} \mathrm{Nickel}$, corona discharge, and secondary electrospray ionization sources with ion mobility-mass spectrometry. Talanta 107, 225232 (2013)

12. Langejuergen J, Allers M, Oermann J, Kirk AT, Zimmermann S: High kinetic energy ion mobility spectrometer: quantitative analysis of gas mixtures with ion mobility spectrometry. Anal. Chem. 86, 7023-7032 (2014)

13. Langejuergen J, Allers M, Oermann J, Kirk AT, Zimmermann S: Quantitative detection of benzene in toluene- and xylene-rich atmospheres using high-kinetic-energy ion mobility spectrometry (IMS). Anal. Chem. 86, 11841-11846 (2014)

14. An Y, Aliaga-Rossel R, Choi P, Gilles J-P: Development of a short pulsed corona discharge ionization source for ion mobility spectrometry. Rev. Sci. Instrum. 76, 85105 (2005)

15. Kirk AT, Grube D, Kobelt T, Wendt C, Zimmermann S: A High Resolution High Kinetic Energy lon Mobility Spectrometer Based on a Low-Discrimination Tri-State Ion Shutter. Analytical chemistry, in press (2018)

16. Kirk AT, Allers M, Cochems P, Langejuergen J, Zimmermann S: A compact high resolution ion mobility spectrometer for fast trace gas analysis. Analyst 138, 5200-5207 (2013)

17. Kirk AT, Zimmermann S: An analytical model for the optimum drift voltage of drift tube ion mobility spectrometers with respect to resolving power and detection limits. Int. J. Ion Mobil. Spec. 18, 129-135 (2015) 
